\title{
La Gloire du peintre et les errements de l'Académie : des pistes pour l'étude des marines de Claude-Joseph Vernet au Salon
}

\section{Nicolas Gaudreau}

Volume 27, numéro 1-2, 2000

URI : https://id.erudit.org/iderudit/1069724ar

DOI : https://doi.org/10.7202/1069724ar

Aller au sommaire du numéro

Éditeur(s)

UAAC-AAUC (University Art Association of Canada | Association d'art des universités du Canada)

ISSN

0315-9906 (imprimé)

1918-4778 (numérique)

Découvrir la revue

Citer cet article

Gaudreau, N. (2000). La Gloire du peintre et les errements de l'Académie : des pistes pour l'étude des marines de Claude-Joseph Vernet au Salon. RACAR : Revue d'art canadienne / Canadian Art Review, 27(1-2), 74-86.

https://doi.org/10.7202/1069724ar

\section{Résumé de l'article}

Thomas Crow (1985) has established that the Academy's Salon was the theatre of an art crisis in the France of Louis XV. This has inspired a comparison between the tempests and shipwrecks of Claude-Joseph Vernet (1714-1789) and his series Les Ports de France (1754-1765), commissioned on behalf of the King by Marigny. In the first part of the article, we examine the popularity of the former paintings. After analysing the enthusiastic response of Diderot in his Salons, directly inspired by Burke's treatise on the sublime, we show that the success of these paintings was in their power to produce strong emotions in the spectator's mind. We then describe the perception of the sea in the eighteenth century in order to anchor Diderot's critique in a body of non-artistic representations. The popularity of Vernet's tempests and shipwrecks rests on a lack of social symbolism. This gives them a universal character with which, unlike the more aristocratie history painting, spectators of ail origins can identify. The last section deals with Les Ports de France. We begin by discussing the choice of landscape for such a prestigious work, which can be explained by the necessity to renew the image of the state. The choice of the seaport motif answered both the need of propaganda by imposing a unitary vision of France and the wish to recall Colbert's heritage. Finally we analyse how the King and the Salon received the series by concluding that it was ultimately a failure for political and aesthetic reasons.
Tous droits réservés @ C UAAC-AAUC (University Art Association of Canada | Association d'art des universités du Canada), 2003
Ce document est protégé par la loi sur le droit d'auteur. L'utilisation des services d'Érudit (y compris la reproduction) est assujettie à sa politique d'utilisation que vous pouvez consulter en ligne.

https://apropos.erudit.org/fr/usagers/politique-dutilisation/ 


\title{
La Gloire du peintre et les errements de l'Académie: Des pistes pour l'étude des marines de Claude-Joseph Vernet au Salon
}

\author{
Nicolas Gaudreau, Univiritite CONCORDIA
}

\begin{abstract}
Thomas Crow (1985) has established that the Academy's Salon was the theatre of an art crisis in the France of Louis XV. This has inspired a comparison between the tempests and shipwrecks of ClaudeJoseph Vernet (1714-1789) and his series Les Ports de France (17541765), commissioned on behalf of the King by Marigny. In the first part of the article, we examine the popularity of the former paintings. After analysing the enthusiastic response of Diderot in his Salons, directly inspired by Burke's treatise on the sublime, we show that the success of these paintings was in their power to produce strong emotions in the spectator's mind. We then describe the perception of the sea in the eighteenth century in order to anchor Diderot's critique in a body of non-artistic representations. The
\end{abstract}

popularity of Vernet's tempests and shipwrecks rests on a lack of social symbolism. This gives them a universal character with which, unlike the more aristocratic history painting, spectators of all origins can identify. The last section deals with Les Ports de France. We begin by discussing the choice of landscape for such a prestigious work. which can be explained by the necessity to renew the image of the state. The choice of the seaport motif answered both the need of propaganda by imposing a unitary vision of France and the wish to recall Colbert's heritage. Finally we analyse how the King and the Salon received the series by concluding that it was ultimately a failure for political and aesthetic reasons.
L fait partie de ces artistes qui furent célébrés de leur vivant, mais que personne ou presque ne connaît plus aujourd'hui. Ainsi, lorsqu'on cite son nom dans la masse d'écrits portant sur le Salon de l'Académic royale de peinture et de sculpture au dixhuitième siècle, Vernet semble évoluer en marge de l'histoire de l'art actuelle. Certes l'admiration que lui vouait Diderot n'échappe à personne, mais sa présence dans des ouvrages à thèse ${ }^{1}$ où dans des études sur Diderot ${ }^{2}$ reste souvent discrète, presque spectrale. Qu'on attribue ce manque d'intérêt pour son œuvre au fait qu'il s'agit manifestement d'une peinture de genre, difficile à intégrer dans les schèmes d'analyse de la peinture de cette période, ou encore au fait que la part la plus en vue de son corpus ait été consacrée à la mer, un motif négligé par la culture occidentale actuelle ${ }^{3}$, il reste qu'en repoussant une étude approfondie du peintre avignonnais, l'histoire de l'art se prive d'une source riche d'informations sur l'art de cette époque et plus particulièrement sur l'Académie et son Salon. En effet, Vernet fut un acteur important de l'événement parisien, s'y distinguant autant lorsqu'il exposait ses tableaux destinés à une clientèle privée que lorsque, coiffé du chapeau de peintre officiel, il présenta la série des Ports de France. Ce sonc cette série et ses tempêtes et naufrages que nous désirons mettre ici en lumière.

Notre approche de la peinture de Vernet emprunte certaines idées développées par Thomas Crow dans Painters and Public Life in Eighteenth-Century Paris ${ }^{4}$. En décrivant comment une création de l'Académie, le Salon, avait favorisé l'émancipation de la critique artistique et l'apparition d'un public moderne doté d'un jugement artistique autonome, cet auteur a démontré qu'une crise de l'art officiel a frappé la France sous Louis XV, déstabilisant la sacro-sainte hiérarchie des genres artistiques et ébranlant l'économie d'une sphère artistique placée sous l'égide de l'autorité politique. Pour illustrer cette crise qui se déclare au Salon, Crow oppose par exemple le succès sans précédent d'arcistes des genres mineurs tels Chardin et Greuze, qui drainent les foules pendant les expositions de peinture, au désintérêt qui afflige en même temps la peinture d'histoire. Reprenant cette hypothèse d'une crise de l'art officiel à l'époque de Vernet, nous avons analysé successivement des productions privées et publiques du peintre afin de faire ressortir, par une mise en opposition de leurs a priori esthétiques et idéologiques, que le désaveu ne s'adressait pas qu'à la peinture d'histoire, mais aussi à d'autres réalisations soutenues par l'Académie.

Dans cet article qui prendra la forme d'un diptyque, nous chercherons tout d'abord à comprendre la popularité des tempêtes et des naufrages de Vernet au Salon. Pour cela, et tout en admettant que la critique est souvent à la fois le produit et l'agent du succès d'une forme artistique et tout en sachant qu'elle n'est pas nécessairement la voix autorisée du public, nous interrogerons l'admiration de Diderot pour ces marines. Au cours de cette démarche que nous avons voulue proche de l'histoire sociale de l'art, nous ferons référence aux théories artisciques de l'époque et emprunterons à l'histoire des mentalités afin de commenter le développement du goût pour la nature et l'évolution de la pensée esthétique qui en est le corollaire, par exemple dans la conceptualisation du sublime. Et avec toutes les réserves qu'impose l'emploi de ce terme, nous introduirons la question du goût bourgeois, qui trouve une caractérisation possible dans les tempêtes et les naufrages de Vernet tels que décrits par Diderot. Car la peinture de Vernet, bien que cantonnée dans un style conservateur très redevable au Lorrain et à ses émules, répondait à des attentes nouvelles sur les plans esthétique et idéologique.

Dans un second temps, l'insistance de Crow sur l'incapa- 
cité de l'Académie à réformer son programme pour reprendre l'initiative de la production artistique nous a inspiré une analyse de la commande des Ports de France. Cette série de quatorze tableaux de grand format, réalisée par Vernet de 1754 à 1765 , constitue un cas exemplaire des efforts déployés par l'institution pour se renouveler tout en ne répudiant pas son rôle central dans la propagande étatique. Après avoir démontré comment cet ensemble, qui constitue la seule commande publique de l'artiste et la plus importante proposée à un peintre sous Louis $\mathrm{XV}$, confere une dimension symbolique et politique à l'espace portuaire, nous tenterons enfin d'établir que ce revirement stratégique de l'Académie, qui renonce pour un temps au genre historique afin de s'attacher les services d'un peintre unanimement apprécié, ne produit pas les résultats escomptés, le Vernet propagandiste ne parvenant pas à supplanter celui qui émeut Diderot.

La représentation de la mer sera donc le fil conducteur de cet article où elle prendra tour à tour des significations esthétiques, culcurelles et politiques. Avec Vernet, il ne s'agit pas de livrer un chapitre de l'histoire de la peinture de marine en France au dix-huitième siècle, qui serait fondé sur la recherche d'une quelconque originalité formelle dans son œuvre, mais plutôt de montrer une fois de plus que l'innovation stylistique ne saurait être le critère déterminant de l'histoire de l'art.

Le paysage bourgeois: Les tempêtes et les naufrages de Vernet

Pendant que les natures mortes de Chardin et les scènes de genre de Greuze attirent les foules des expositions d'art, les tempêtes et les naufrages de Vernet goûtent aussi à la popularité que connurent les genres mineurs au dix-huitième siècle. On en trouve une preuve éloquente dans les Salons de Diderot, qui couvrent cette exposition bisannuelle de 1759 à 1781, et où l'auteur illustre sa pensée esthétique à l'aide des œuvres de Vernet. C'est en effet dans le Salon de 1767, au cours de sa fameuse "Promenade Vernet" 5 , que Diderot établit les premiers rapprochements entre les tempêtes et les naufrages du peintre et la catégorie esthétique du sublime. Sur cet aspect des tempêtes et des naufrages de Vernet, Diderot emprunte l'essentiel de son propos théorique au traité d'Edmund Burke ${ }^{6}$ en faisant de la mer la cause ultime du sublime. Dans le Salon de

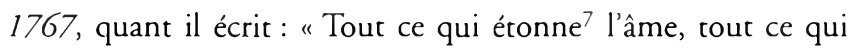
imprime un sentiment de terreur conduit au sublime $n^{8}$, puis, "une vaste plaine n'étonne pas comme l'océan ", il s'approprie littéralement ces paroles de Burke: "Whatever is in any sort terrible, ... or operates in a manner analogous to terror, is a source of the sublime ${ }^{10}$, et, "A level plain of a vast extent on land, is certainly no mean idea; the prospect of such a plain may be as extensive as a prospect of the ocean; but can it ever fill the mind with any thing so great as the ocean itself? This is owing to several causes, but it is owing to none more than this, that the ocean is an object of no small terror. "11 Mais alors que Burke présente l'étendue immense et le caractère terrible comme deux attributs du sublime océanique, Diderot semble ne retenir que le deuxième en ne considérant que la dimension dramatique des tempêtes et des naufrages de Vernet. Chez le critique, le sublime s'associe en effet à la terreur et à une forme d'impuissance humaine devant une manifestation des forces hostiles de la nature. Voilà ce qui explique pourquoi il accorde tant d'importance à l'aspect humain de cette peinture et pourquoi il en exacerbe le côté tragique.

Pour mieux comprendre cette distinction, il faut jeter un coup d'œil à la stratégie narrative employée par le critique lorsqu'il est question des naufrages de Vernet (fig. 1). Chaque fois Diderot respecte un plan similaire : s'attardant d'abord à la description du lieu et au déferlement d'énergie, il dépeint ensuite les effers de ce dernier sur les bateaux et termine en s'apitoyant sur le sort des naufragés. Dans le Salon de 1765, il conseille le lecteur de cette façon: "C'est surtout aux figures qu'il faut s'attacher. Le vent est terrible, les hommes ont peine à se tenir debout. Voyez cette femme noyée qu'on vient de retirer des eaux, et défendez-vous de la douleur de son mari, si vous le pouvez. "12 Couvrant le Salon de 1763, il décrivait longuement les effets funestes d'une tempête sur un groupe de malheureux. En 1767, enfin, pour metcre un terme à une autre description ${ }^{13}$, il avouait théâtralement : "Je voyais toutes ces scènes touchantes et j'en versais des larmes réelles. " ${ }^{14}$ Sa conclusion se conforme bien sûr au dénouement prévisible d'un naufrage, mais le caractère emphatique de la finale montre bien le genre d'impact que la scène a sur le critique. Ce n'est donc qu'en s'associant aux personnages malheureux représentés dans le tableau, et en éprouvant de la pitié, que Diderot est à même de prendre la mesure des forces cataclysmiques et d'expérimenter l'émotion sublime ${ }^{15}$.

La forte charge émotionnelle suscitée par les naufrages de Vernet semble donc fasciner le philosophe. Ainsi, quoiqu'en dise E. M. Bukdahl ${ }^{16}$ et malgré ce que Diderot lui-même a écrit sur les vertus pédagogiques de l'art de Greuze, accordant par le fait même une fonction utilitaire à sa peinture, nous concluons que la réponse de Diderot aux marines de Vernet reste principalement confinée au registre de l'émotion. Nous préférons voir dans les tempêtes et les naufrages de Vernet l'expression d'un sublime pathétique qui affecte le critique en le faisant témoin de l'action dévastatrice de la mer déchainée sur l'humanité fragile. Ce sublime s'apprécierait qualitativement par la réponse émotionnelle qu'il suscite chez le spectateur, une réponse qui passe par l'association avec les victimes du drame et dont les critiques des Salons de Diderot offriraient les meilleurs exemples.

Si Diderot puise allègrement dans le texte de Burke, il ne s'en fait pas pour autant le traducteur servile. Par exemple, 


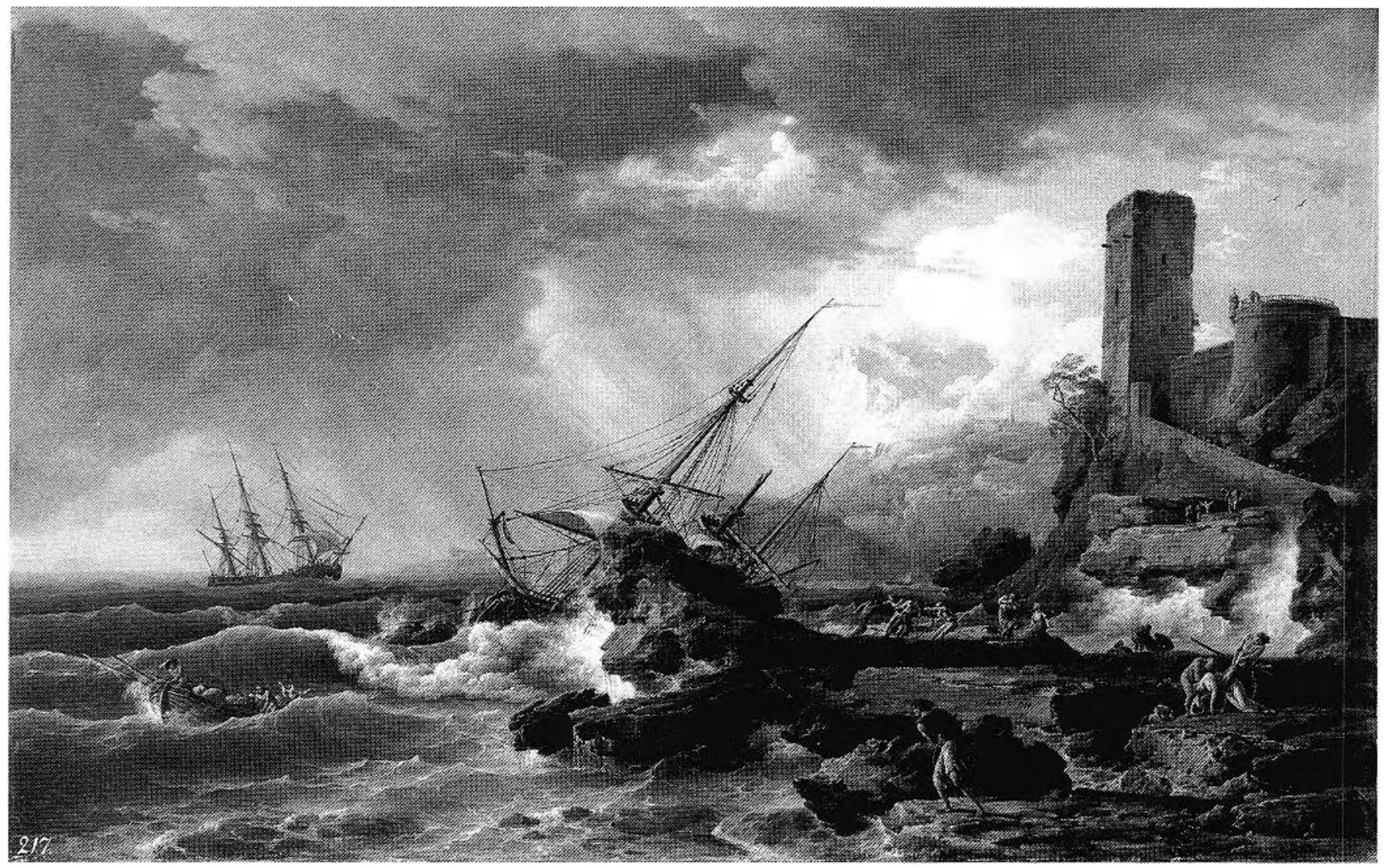

Burke parle de la vraie mer dans son traité alors que le philosophe français, lui, s'émeut devant son interprétation artistique. Cette distinction est essentielle puisqu'elle repose sur une conception différente de l'art. Comme l'écrit Bukdahl : "Dideror ne peut accepter l'affirmation de Burke selon laquelle les événcments de la vie réelle exercent toujours une influence plus profonde que l'interprécation artistique qui en est donnée. "17 $^{17}$ Réfléchissant sur cet aspect de la pensée esthétique de Diderot. Herbert Dieckmann conclut que si "Diderot a hérité de la doctrine classique le principe de l'imitation de la nature ${ }^{18}$, il lui a accordé de multiples significations suivant une évolution que l'historien présentc comme allant de l'imitation à l'expression. En fait, Diderot avait bel et bien adopté le sublime de Burke, mais en l'intégrant à une pensée qui accordait à l'art une plus grande autonomie parce qu'elle ne reconnaissait pas la supériorité classique du modèle sur l'image.

En face de la critique emportée de Diderot, il est difficile de distinguer la part de sincérité de celle qui tient du procédé littéraire; nous pensons malgré tout qu'elle témoigne d'un état d'esprit propre à son siècle face aux choses de la mer. Pour explorer cette hypothèse, nous déborderons provisoirement du cadre de l'histoire de l'art pour nous pencher sur la perception de la mer au dix-huitième siècle. Ce retour au référent peut sembler paradoxal, mais il faut comprendre qu'au moment où l'esthétique manifeste une volonté de s'affranchir du réel, elle se construit toujours sur des critères qui s'appliquent indifféremment à l'art et à la réalité. ${ }^{19}$

La mer au dix-huitième siècle en France et en Angleterre: De la peur à l'apprivoisement

Avant d'entamer cette parenthèse, nous devons souligner le fait que Vernet n'est pas le seul artiste de son temps à employer l'image agressive de la mer à des fins artistiques. S'il fait figure d'exception dans une France qui préféra suivre Rousseau à la montagne avant que Bernardin de Saint-Pierre ne lui indique le chemin des $\operatorname{côtes}^{20}$, la situation est tout autre en Angleterre. Outre-Manche, où Vernet compte de nombreux admirateurs et clients, la littérature explore ce riche filon qui donne lieu à d'immenses succès d'édition. Que ce soit sous la forme exotique décrite par Defoe ${ }^{21}$, ou celle plus journalistique mais tout aussi spectaculaire des récits de naufrages ${ }^{22}$, le public anglais raffole en effet des accès de rage de l'océan.

Au dix-huitième siècle, la peur de la mer reste très présente 
en Europe: "Jusqu'aux victoires de la technologie moderne, la mer était associée dans la sensibilité collective aux pires images de détresse. Elle était liée à la mort, à la nuit, à l'abîme. " ${ }^{23} \mathrm{En}$ Angleterre, nous rappelle T.S.R. Boase, "of all contemporary nightmares that of the shipwreck was one of the most obsessing $" 24$. Dans une histoire de la mer qui, comme l'a établie Alain Corbin, concerne surtout la zone littorale en ce que la présence humaine y fut plus marquée et marquante ${ }^{25}$, la tempête et le naufrage offrent sans conteste les images les plus fortes, avec peut-être celles, plus récentes, des profondeurs abyssales. Tour à tour négligé, évité ou convoité, le rivage émerge comme un lieu investi de significations multiples. Suivons-y Corbin.

Pendant tout le siècle qui vit naître et mourir Vernet, la mer suscita un mélange de crainte et de dégoût, provoquant un malaise entretenu par une certaine ignorance ${ }^{26}$ et la survivance de visions millénaires où l'océan était perçu comme un "instrument de punition $n^{27}$. Parmi les sources classiques toujours bien vivantes de cette perception, la mer déchaînée par la colère des dieux durant la guerre de Troie, les voyages d'Ulysse de même que la tempête de Virgile entretenaient la méfiance envers l'étendue d'eau. Autre modèle obligé, le récit biblique du Déluge contribuait peut-être davantage à cette peur toujours présente dans la tradition judéo-chrétienne : l'idée de la montée inexorable des eaux transformant la terre en un enfer aquatique provoquait en effet l'angoisse insurmontable de l'engloutissement.

Ces symboliques toutes puissantes avaient pour conséquence de retarder l'appréciation esthétique de l'espace maritime. En effet, l'étendue illimitée de l'océan, la côte rocheuse déchiquetée tout autant que la plage informe, contredisaient les critères classiques de la beauté que sont l'unité, l'harmonie et la clarté. Moins dissimulé aux regards que la pleine mer et considéré comme le vestige visible du déluge, le rivage constituait à cet égard "le plus effroyable spectacle offert par la nature "28. Quant à la surface océanique, son apparente vacuité et sa capacité d'absorption infinie en faisaient l'antithèse du jardin puisque l'homme ne pouvait y imprimer sa marque, ce qui, nous suggère Corbin, expliquerait son absence de l'Arcadie et de l'Éden primitifs ${ }^{29}$. Et comme les côtes désertes étaient aussi le lieu de l'invasion armée et de l'arrivée de la peste, le repaire et le terrain d'action des naufrageurs, des risques réels s'ajoutaient aux mythes.

Plus tard, malgré les coups d'éclat des explorateurs de la Renaissance qui bousculèrent les perceptions de telle sorte qu'une minorité de gens put contempler la mer d'un œil plus conquérant, la méfiance et la peur continuèrent de prévaloir lorsqu'il s'agissait de la rendre en images. On se souvient qu'en Hollande, dans une société qui devait sa richesse et sa puissance à la mer, on avait souvent fait appel au côté terrible de l'océan pour construire des exemples de conduite morale ou politique. ${ }^{30}$ Aux légendes et aux histoires retransmises depuis plus de deux millénaires, il faudrait ajouter que le domaine marin avait toujours conservé un statut juridique indéterminé, ce qui attisait les convoitises. Il y avait bien eu, en France, cet édit royal de 1681 qui, tout en visant à asseoir l'autorité du gouvernement central sur un domaine extrêmement agité, garantissait la propriété des restes d'un bateau échoué et des biens éparpillés à son possesseur original. Mais, faute d'effectifs de police suffisants, dans plusieurs régions le droit d'épave ou de bris se révélait inapplicable et un naufrage se concluait souvent par un pillage. Prétextant de leur grande pauvreté ou se réclamant du droit seigneurial, il arrivait en effet que les habitants des côtes confisquent ou volent les objets dispersés en ajoutant à la détresse des naufragés. ${ }^{31}$

Du vivant de Vernet, on observe un lent apprivoisement du domaine marin. Accumulant les initiatives, le touriste éclairé et l'homme commerçant poursuivent l'entreprise de démystification des mers lancée par les explorateurs de la Renaissance. L'institutionnalisation du Grand Tour illustre bien ce désir plus généralisé de découvrir le monde, mais les premiers touristes s'intéressent plus aux vestiges culturels qu'au spectacle de la nature. ${ }^{32}$ Il faut avouer que le voyage par bareau, long, inconfortable et soumettant les passagers au terrible mal de mer, laissait peu de place au plaisir des yeux.

Dans l'univers capitaliste en pleine expansion, la marine marchande prenait toute son importance pour qui voulait récolter la manne du commerce des produits exotiques ou de l'industrie militaire. L'Angleterre était la plus avancée dans cette course. Exposé par son insularité au risque permanent d'une domination étrangère, le royaume n'avait d'autre choix que d'appuyer son industrie foisonnante sur une vaste structure de transport maritime. Dans ce pays, la mer constituait même un facteur d'identité et le bateau était à la fois le symbole de la richesse, de l'ouverture sur le monde et de la sécurité nationale. La France, une autre puissance coloniale établie, accusait un recul face à son ennemi traditionnel. Politiquement, et cela malgré les ambitions et les efforts de Colbert au siècle précédent, elle semblait guidée par une atcitude opportuniste et manquait d'une vision d'ensemble dans ses entreprises maritimes, ce qui n'empêchait certes pas le commerce par bateau d'assurer la fortune de nombreuses villes côtières. Il reste que malgré les progrès constants de la navigation ${ }^{33}$, l'armement d'un navire en n'importe quel pays comportait des risques qui enrichissaient les compagnies d'assurances.

Comme Jean Ehrard le montre dans son ouvrage L'Idée de nature $^{34}$, les hommes de science ont beaucoup contribué à l'effort de démystification de la mer en s'attachant à l'observation et à la description de ses caractères physiques et biologiques. En vertu de cette attention, ils conféraient au territoire côtier une valeur positive, voire une certaine noblesse qui venait contredire les perceptions négatives encore bien ancrées. Mais 
au sein de la communauté scientifique, c'est peut-être davantage aux médecins que l'on doit l'évolution des mentalités.

Face à la montée d'angoisse qu'entraînaient la frénésie des agglomérations urbaines et les incerticudes nouvelles du capitalisme, plusieurs personnes fortunées quittaient la ville quand elles en avaient l'occasion. On se dirigeait le plus souvent vers une résidence à la campagne; mais alors qu'en France, la montagne restait la destination privilégiée des amateurs de nature sauvage, en Angleterre, il arrivait de plus en plus fréquemment que l'on se retrouve sur la côte. Suivant leur instinct ou les conseils de leurs médecins, les membres de la gentry s'y rencontraient dans un nouveau cadre de sociabilité pour combattre le spleen et autres vagues à l'âme. C'est dans ce contexte que les plus hardis des deux sexes se livraient à la pratique insolite du bain de lame, une extrapolation de la coutume plus ancienne du bain froid. Encouragé par les vertus bienfaitrices de l'eau glaciale ${ }^{35}$ et salée, on affrontait les vagues en plongeant sous le regard du maître de bain et selon un rituel organisé. Amplifié par la crainte, le double choc causé par l'eau froide et le manque d'air devait, en secouant l'âme, libérer de l'angoisse, combattre la stérilité, prolonger la longévité et amener tous les bienfaits découlant de ce que l'on pourrait appeler une thérapeutique du sublime. ${ }^{36}$ Cette pratique, qui précédait et annonçait la villégiature en bord de mer telle que nous la connaissons aujourd'hui, était bien sûr le lot d'une minorité privilégiée; elle démontre cependant que la mer restait encore à domestiquer et que sa fréquentation ne se faisait pas toujours sur un mode spontané. En fait, le bain de lame avait une nature paradoxale puisqu'en fondant son efficacité médicale sur la peur de l'eau, il faisait d'un aspect négatif une incitation à la découverte: "En jouir, éprouver la terreur qu'elle inspire tout en désarmant ses périls : telle sera la stratégie de la villégiature maritime. " ${ }^{37}$

$\mathrm{Si}$ on découvrit lentement la mer au dix-huitième siècle, ce fut donc avant tout pour des raisons utilitaires, indépendantes de toutes considérations esthétiques. Des poètes ont certes chanté sa beauté, mais ce fut le plus souvent sur un ton convenu. Peu à peu cependant s'y greffa le plaisir de la contemplation; on peut toutefois affirmer que, d'une manière générale, on navigua sur l'eau et l'on s'y jeta avant que de l'admirer. Ex si la plus grande partie de la population n'avait en fait qu'une connaissance limitée, voire nulle, de la mer, cette dernière avait une présence forte dans l'imaginaire et constituait une source d'images puissantes qui allaient contribuer à déstabiliser le modèle classique de la nature.

Le sublime, catégorie esthétique participante de l'idéologie bourgeoise ${ }^{38}$

Décrire la perception de la mer au dix-huitième siècle nous a permis d'ancrer la critique "terrorisée " de Dideror dans un ensemble de représentations débordant le domaine de l'esthétique. Toutefois, ce seul rapprochement ne saurait expliquer la popularité des tempêtes et des naufrages de Vernet au Salon. Répondre à cette question revient à se demander ce qui distingue les thèmes de Vernet de la peinture d'histoire, le genre favorisé par l'Académie conformément à ses préceptes hiérarchiques $^{39}$.

Revenons aux naufrages de Vernet. Aucun ne se déroule en pleine mer, mais près d'une côte déchiquetée et rocheuse et le point de vue se trouve sur la terre ferme ${ }^{40}$. Dans ce paysage qui prend l'allure d'un théâtre à ciel ouvert, le naufrage se mue en un spectacle offert aux regards, un drame joué sur une scène qui permet à Vernet de toucher doublement son public, d'abord en ayant recours à de nombreux personnages pour évoquer une vaste gamme d'émotions, ensuite en situant l'action dans un décor qui rejoint les références littéraires et historiques de ses contemporains. Mais il y a plus que cela dans la fascination d'un Diderot pour ces tempêtes et ces naufrages. Nous croyons en effet que l'engouement pour les naufrages de Vernet repose sur un principe de reconnaissance et d'identification. Sans vouloir reconduire la pensée presque manichéenne d'un Arnold Hauser, qui distingue trop catégoriquement la bourgeoisie de l'aristocratie, on sait que ce qu'on a appelé l'art bourgeois remportait alors un vif succès parce qu'une certaine partie de la population pouvait s'y reconnaître. En se voyant représentée dans la peinture moralisante d'un Greuze ou dans les romans de Richardson, par exemple, une couche de la société accédait à une existence artistique qui confortait son désir de légitimité politique. D'une certaine façon, nous pensons qu'un mécanisme semblable est à l'œuvre dans les scènes de Vernet, mais d'une manière plus large encore puisque l'émotion sublime provoquée par le naufrage peint possède cette propriété d'être immédiatement accessible à tout spectateur, quel que soit son milieu d'origine. En plus de se dérouler dans un lieu sans connotation sociale, les naufrages de Vernet taisent le nom des navires en perdition. En réduisant les vêtements des naufragés à des lambeaux, ils préservent aussi l'anonymat de ces derniers de sorte qu'il est difficile d'en connaître le rang social. Par ces procédés, l'artiste facilite l'identification de tout spectateur aux personnages du tableau et fait du naufrage un événement universel, rejoignant en cela les critiques du pouvoir établi. Sans s'encombrer de la leçon de conduite, de l'épisode mythologique ou de la célébration d'un chef politique qui caractérisent les œuvres plus lourdement chargées de sens, les naufrages de Vernet arrivent à toucher le cœur de tous leurs publics en déclenchant le processus d'identification que nous avons décrit chez Diderot. C'est donc la dégradation des signes d'appartenance sociale qui autorise cette identification, et non la stigmatisation par une marque de classe quelconque. Et peu importe que la majorité des visiteurs du Salon n'ait eu en réalité qu'une connaissance limitée, 
Figure 2. Claude-Joseph Vernet, L'Intérieur du Port de Marseille, vû [sic] du Pavillon de l'Horloge du Parc, 1754, huile sur toile, $165 \times 263 \mathrm{~cm}$, Paris, Musée de la Marine (crédit photographique : (C) Musée de la Marine).

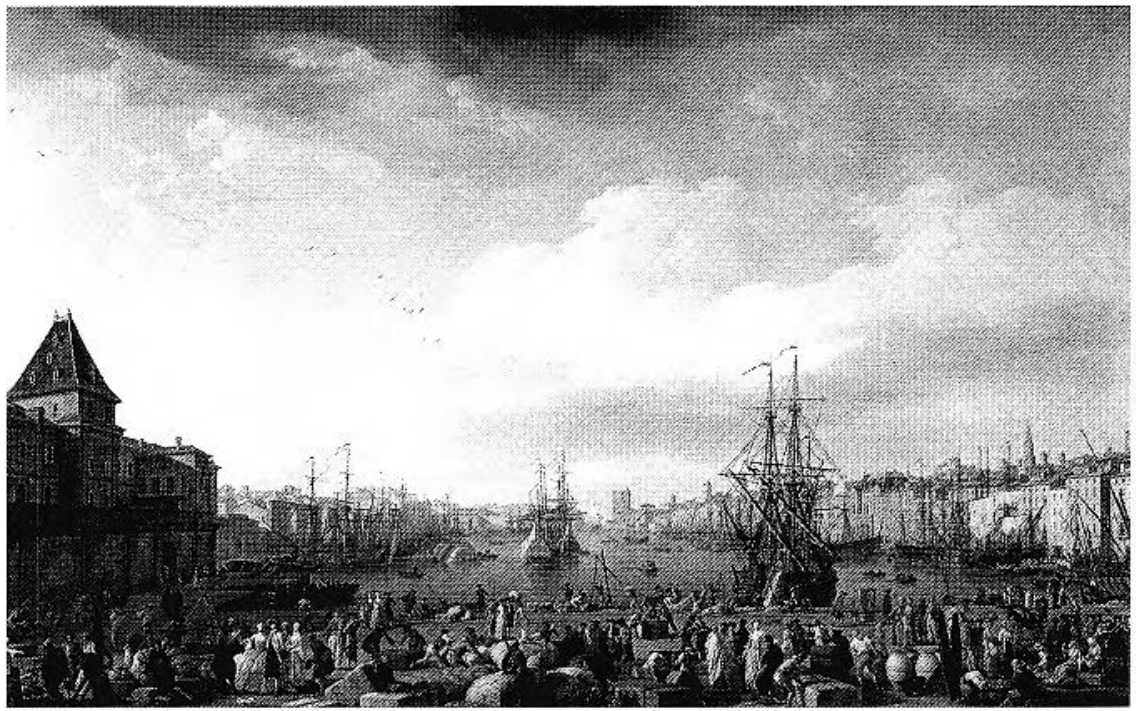

voire nulle, de la vraie mer, la portée du référent, qui rejoint les perceptions des contemporains, et la force du réalisme ${ }^{41}$ suffisent à susciter l'identification qui fait du naufrage un acte de la tragédie bourgeoise : anonyme et hors de l'histoire.

La peinture bourgeoise de Vernet participerait donc d'un art qui, tout en s'appropriant la dimension tragique et le pathos habituellement réservés à la peinture d'histoire, reste immédiatement accessible sur le plan de l'émotion parce qu'il ne s'alourdit pas de références littéraires ou historiques. Face à un concept du beau trop empêtré dans les règles du bon goût et les conventions, le sublime pathétique des naufrages de Vernet confere à l'appréciation subjective de l'art sa pertinence moderne. Bien sûr, il ne s'agit pas d'une peinture délibérément politique ou militante, mais d'une production commerciale qui vise le plus large marché possible. ${ }^{42}$ En partageant avec les aristocrates la délectation offerte par les tableaux de Vernet, le public diversifié du Salon obtient quant à lui une légitimité critique et une place nouvelle dans la sphère artistique. Une place qu'il ne manquera pas de revendiquer au point de se l'approprier par la suite.

\section{Le paysage monarchique : Les Ports de France}

La première partie de cet article concernait ce qu'on peut appeler le marché privé de l'art. Risquons maintenant une incursion du côté de l'art officiel où d'autres marines de Vernet, la série des Ports de France, ont obtenu une grande visibilité (fig. 2). On peut se demander pourquoi, vers la moitié du dix-huitième siècle, la représentation de ses principaux ports de mer devient une priorité de l'État français, au point d'en faire une des plus grandes commandes du règne de Louis XV. Les archives disponibles se montrant muettes sur les motivations exactes du mar- quis de Marigny, maître d'œuvre et principal interlocuteur de Vernet pendant la durée du projet, nous tenterons de démontrer qu'un tel parti découle d'impératifs politiques autant qu'esthériques. La commande des Ports arrive en effet à un moment particulier de l'histoire de l'art officiel français. Elle s'inscrit dans le contexte d'une crise que Thomas Crow relie à la mouvance de la guerre de Sept Ans et à l'évolution que subit la pensée artistique face à l'importance croissante du Salon annuel de l'Académie (où, comme nous l'avons dit, Vernet est un acteur de premier plan). Nous verrons que l'œuvre de Vernet porte les stigmates de cette crise et qu'elle fournit une illustration supplémentaire de la thèse de Crow.

Les circonstances exactes de la commande restent floues. Traditionnellement, on a reconduit l'interprétation de Léon Lagrange ${ }^{43}$ selon laquelle Vernet fut rappelé de son exil italien pour entrer au service du roi de France, encouragé en cela par le surintendant des bâtiments du roi et frère de la marquise de Pompadour, le marquis de Marigny. Plus de cinquante ans après Lagrange et suite à la découverte d'une lettre inconnue jusqu'alors, Florence IngersollSmouse a suggéré que la situation de plus en plus précaire du beau-père de Vernet dans l'entourage du pape aurait pu inciter le peintre avignonnais à quitter lui aussi la ville éternelle. ${ }^{44}$ Mais peu importent les véritables raisons de ce départ, Vernet entreprend son périple en 1754 , soit une année après son retour d'Italie.

L'interruption de la commande eut lieu en 1765, environ deux ans après la signature avec les Anglais d'une paix peu réjouissante qui confirmait la domination britannique sur l'Atlantique et sur les colonies américaines. Ces circonstances affaiblissaient fortement toute propagande axée sur le contrôle d'une mer que les dirigeants préféraient oublier momentanément. L'immense effort de guerre avait grevé pour un temps l'économie française et le trésor royal à sec ne pouvait plus soutenir les largesses du mécénat culturel. En réalité, le périple de Vernet ne s'arrêta pas brusquement, mais la lassitude de l'artiste et de Marigny devant la tâche qui restait à accomplir recouvrit progressivement le projet d'un voile d'indifférence. Le peintre ne souffrait plus les rigueurs du voyage et les retards de paiement. Ayant reçu ses appartements au Louvre, Vernet s'y installa pour se consacrer de nouveau au lucratif marché privé. Enfin, le quinze avril 1764, la mort de la marquise de Pompadour dut contribuer fortement à la fermeture définitive du dossier. Bien que Marigny ait conservé son poste jusqu'à la venue de Louis $\mathrm{XVI}$, son influence diminua progressivement après la dispari- 
tion de sa sœur. Avec celle qui avait fait du soutien des arts une priorité politique, s'éteignaient les derniers espoirs de voir un jour se terminer une commande qui comptera finalement quatorze tableaux sur les vingt-quatre initialement prévus.

A l'époque de la série des Ports, la peinture d'histoire, principal véhicule de l'image de la monarchie, connut une forme d'éclipse. Devant le rejet qui marquait le haut genre, critiqué pour son pédantisme, son iconographie hermétique et son éloignement du peuple, et devant la faveur que goûtaient des peintres moins galonnés, la direction des bâtiments réduisit le budget consacré aux tableaux d'histoire. Bénéficiant de la bienveillance de Marigny, qui leur donnait pour ainsi dire le champ libre, et gratifiés de la caution que leur apportait l'admiration du public et des critiques du Salon, des peintres comme Greuze et Verner occupèrent une grande place sur la scène artistique officielle, plus grande en fait que celle qu'on avait jamais vu occupée par des artistes spécialisés dans les genres secondaires. C'est donc dans l'espoir de comprendre comment l'Académie entendait se sortir de son impasse que nous chercherons les motivations de l'entreprise des Ports.

Premièrement, on peut se demander ce qui a pu amener la valorisation du paysage à ce moment précis de l'histoire de l'art monarchique français. Cette apparition marque en effet une rupture dans une tradition iconographique fonctionnant sur le mode allégorique et centrée sur la personne royale. Dérivée des modèles impériaux romains et de la figure christique, la propagande avait jusque-là fondé le pouvoir terrestre du monarque sur sa double nature physique et métaphysique. ${ }^{45}$ En faisant reposer la domination sur un ascendant religieux retransmis par le sang, on avait assuré la pérennité de la lignée royale et justifié ses prérogatives. Le même héritage de l'art chrétien se voyait aussi détourné pour faire du corps du roi la métaphore de la nation, une stratégie qui culmina dans l'art de la cour de Louis $\mathrm{XIV}$, où cette association devait trouver sa formulation essentielle dans la phrase célèbre et apocryphe: "L'État c'est moi. "

Même si la tradition "régicentrique " 46 perdure jusqu'au siècle de Louis XV, la métaphore qui lie le roi et l'État commence à se disloquer en même temps que l'idée de nation progresse. Ainsi, David Castriota écrit :

\footnotetext{
The political trends of the modern period are characterized by a gradual but steady shift towards a more collective concept of the state as an amalgam of individual enterprise and achievement that no one man could encompass or epitomize, and a concept that is progressively less overt or insistant about the role of this divinity as the ultimate source of the success of the state. ${ }^{47}$
}

Cette évolution de la conception de l'État a évidemment des répercussions sur sa représentation. En effet, avec ce qu'on peut appeler la sécularisation du pouvoir, la baisse de la puissance symbolique de la personne physique du roi peut donner lieu à un bouleversement iconographique en faveur du paysage.

Il n'est pas nécessaire d'attendre l'avènement de la république ou de la démocratie moderne pour assister à ce changement. Dans Landscape and Power, W.J.T. Mitchell écrit que tôt ou tard dans une société d'envergure impériale, comme c'est le cas de la France du dix-huitième siècle ${ }^{48}$, un art du paysage fait son apparition. Dans les états exerçant leur autorité sur d'immenses territoires qui ne sont jamais parcourus en totalité par les ressortissants de la métropole, cette apparition serait facilitée parce que, de l'avis de Mitchell, le référent paysage a ceci de particulier qu'avant même d'être sublimé par l'acte artistique, il est déjà une représentation : "What we tend to forget, however, is that this subject matter [le sujet de la peinture de paysage] is not simply raw material to be represented in paint but is already a symbolic form in its own right. " ${ }^{49}$ Signe autotélique, le paysage naturel ou domestiqué renvoie bien sûr à lui-même, mais en lui s'accumulent aussi des significations sociales et politiques, au point que Mitchell y voie, dans une allusion à Marx, un "social hieroglyph "50. Comme l'inaccessible roi dans l'imaginaire de son peuple, le paysage réel serait alors un signe iconique propice au détournement idéologique.

Avec les Vues des ports, la peinture de paysage obtient donc la reconnaissance officielle et se fait le lieu d'un message politique. Dans ce contexte particulier qui précède la guerre de Sept Ans, la représentation du port devient même une priorité nationale, un choix qui prévaut sur les autres motifs disponibles dans la topographie diversifiée de la France, à cause bien sûr de la lutte militaire qui l'oppose à l'Angleterre pour le contrôle des colonies. Pourtant ceux qui seraient tentés de voir dans ce programme un plaidoyer en faveur d'investissements dans la marine de guerre ne doivent pas oublier que les neuf ports représentés par Vernet n'ont pas tous une véritable importance stratégique. Et même si l'on considère le rôle économique comme une partie intégrante de l'effort de guerre, il faut comprendre que les ports de Sète ou de Bandol, par exemple, ne souffrent en aucune manière la comparaison avec ceux de Marseille ou de Bordeaux. En fait, nous croyons que la seule justification militaire et coloniale, bien que primordiale, reste incomplète.

La première interprétation que nous souhaitons produire exige d'aborder la série comme un tout, un exercice qui aurait été difficile pour les contemporains de Vernet puisqu'après leur présentation progressive au Salon au fur et à mesure de leur achèvement, les tableaux échouèrent au palais du Luxembourg dans une indifférence presque totale. Pour une série d'une telle ampleur, cette solution improvisée surprend à une époque où les peintres devaient souvent tenir compte de la destination finale de leur travail lors de l'exécution. Dans l'état actuel de nos connaissances, et tout en convenant que la découverte d'un 
document révélateur pourrait venir nous contredire, le mutisme des commanditaires sur leurs intentions décoratives nous incite à penser que cet ensemble est autonome et qu'il trouve sa plénitude sémantique dans son seul regroupement, peu importe le lieu d'exposition.

Nous croyons en fait qu'il faut envisager la série des Ports comme un véritable manifeste géopolitique. Enrichi des ports de Bretagne et de Normandie qui figuraient sur L'Itinéraire (le document précisant le nombre et l'angle des vues), l'ensemble aurait regroupé les plus importants ports de mer français de l'époque. En laissant de côté des ports fluviaux pourtant très actifs comme Lyon sur le Rhône ou Roanne sur la Loire, on dessinait une couronne épousant les limites territoriales de la France et ouverte seulement sur le flanc continental. Ce serait conséquemment pour que la chaine des ports reste ininterrompue sur tout le pourtour côtier qu'on inséra le port mineur de Bandol entre Marseille et Sète. ${ }^{51}$ La signification de l'ensemble tiendrait donc à sa capacité de rapprocher artificiellement dans l'espace de la galerie de peinture les extrémités de "l'étoile " 52 française formée par le réseau des communications émanant de Paris.

Comme les écrits de Fernand Braudel l'ont montré, l'histoire de la France peut s'expliquer par une opposition continue de forces centripètes et centrifuges. Cette histoire raconte la volonté fluctuante du pouvoir central d'effacer les disparités régionales et d'insuffler une identité forte et un sentiment de communauté à cette constellation de pays, de provinces et de hameaux isolés qui vont constituer le territoire national. Pendant des siècles, le développement d'un vaste réseau de transport (routier et fluvial) et l'étirement des tentacules de l'administration n'ont pu que lentement et difficilement venir à bout des disparités de langage, de culture et des indépendances jalouses des différents sous-ensembles géographiques. Encore en 1750, au moment où l'ennemi anglais frappe à la porte et malgré ce "décloisonnement amorcé au début du XVIII siècle ${ }^{53}$, la France reste plurielle et parler d'une identité française tient de l'abstraction. Conséquemment, on peut penser que la commande des Ports s'inscrit dans le vaste mouvement unificateur en dessinant une image de la France axée sur son front de mer et sur les activités qu'elle y déploie.

La représentation des ports constitue une stratégie fort appropriée pour la construction d'une vision unitaire de la France. Tout d'abord, dans sa variante fortifiée et urbanisée, le port signale une occupation et une appropriation radicales du paysage. Son édification procède d'un acte transformateur qui marque la mainmise de la civilisation humaine et qui ne laisse rien à l'indétermination de la nature. Souvent situés sur l'estuaire d'un fleuve navigable ou sur une route importante, les ports les plus actifs sont des plaques tournantes majeures pour tous les types de liaisons et jouent un rôle capital dans la circulation des biens et des personnes. Braudel place d'ailleurs l'évolution des transports suscitée par l'expansion du commerce et les déplacements de soldats en tête des principaux facteurs de la cohésion de la nation française ${ }^{54}$. Dans les régions les plus exposées au risque d'invasion étrangère, ils assument aussi une fonction de vigile et leur conquête donne le plus souvent l'assurance de contrôler toute une région. Une autre particularité notable des ports est leur situation sur la frontière naturelle formée par la ligne côtière, une délimitation beaucoup moins arbitraire et incertaine que les frontières continentales. En effet, lorsqu'on la compare à d'autres frontières naturelles, comme le Rhin, la côte paraît moins précaire puisque, n'étant pas partagée par deux pays limitrophes, elle se trouve peu exposée aux avancées territoriales. Disséminés sur le périmètre du corps principal de la métropole, les ports de mer n'ont pas non plus la précarité des établissements coloniaux. Ils forment donc une ultime frontière en se faisant le point de rencontre du familier et de l'étranger; en quittant la rade à bord d'un navire, l'homme du dix-huitième siècle se retrouve aussitôt dans un espace autre, un ailleurs qui fait rêver et trembler. Pour toutes ces raisons qui touchent à l'économie, à la sécurité nationale et au sentiment géographique, on peut donc considérer la commande des Ports comme une tentative de fixer dans le temps et dans l'espace l'image unitaire d'une France définie par ses limites extrêmes.

En plus de servir l'image d'un état, la propagande répond souvent aux ambitions personnelles de ses initiateurs. En effet, l'artiste, l'homme de lettre ou le ministre qui soumet un projet au monarque le fait souvent pour obtenir sa protection ou assurer ses privilèges. La commande des Ports n'était pas exempte de telles intentions et il est permis de penser que Marigny, en confiant cette œuvre à Vernet, employait une vieille stratégie pour se faire valoir aux yeux de Louis XV. Assurément, il s'efforçait ainsi de rappeler et de s'approprier le souvenir de Colbert, qui encouragea le commerce et la marine pour raffermir la souveraineté de l'État français sur son propre territoire. ${ }^{55}$

Thomas Crow attribue cette tentative d'inscription dans une lignée d'administrateurs hérö̈ques à un désir de reconnaissance sociale. En effet, le geste de Marigny trahit l'inconfort ressenti par cette race de financiers qui, comme lui, doivent leurs titres de noblesse à leur fortune. Crow écrit: "The Lenormands [le clan familial de Marigny] had no choice but to do this in terms of the Academy's established legal mandate; precisely because they were parvenu bourgeois, they had to make their policy to revive the standards and practices of Colbert's half-remembered golden age. " ${ }^{56}$ Malheureusement pour Marigny et ses proches, son entreprise échoua. Il ne toucha ni le roi ni le public du Salon, ratant du même coup les deux cibles qu'il s'était fixées.

Rappelons que la série des Ports fut finalement reléguée au palais du Luxembourg ${ }^{57}$. Même si le cycle de Rubens s'y trouvait encore à l'époque, l'ancien palais de Marie de Médicis 
n'avait plus qu'une fonction subalterne; mal entretenu, il servait à loger des seigneurs de passage à la Cour. Ignorant des lieux plus prestigieux, on choisit donc pour l'exposition finale un endroit de second ordre, suivant une décision qui marque une forme de désaveu. Certes la mer évoquait le mauvais souvenir de la guerre tout juste achevée, certes la série était amputée de plusieurs ports, mais la situation suffit-elle à expliquer la réception froide dont le roi gratifia l'œuvre une fois qu'elle fut arrêtée?58 Pour mieux comprendre cette réaction, il faut de nouveau considérer la commande des Ports comme une œuvre de propagande proposant une certaine vision de l'État français. C'est ici que nous rejoignons Crow en inscrivant l'échec des Ports de France dans une crise de la représentation du pouvoir généralisée.

Tentons d'abord de comprendre ce qui, dans l'œuvre même, a pu rebuter son principal commanditaire. Le parti pris en faveur du réalisme, le souci d'une représentation exacte de la technique navale, l'attention aux détails des costumes et la volonté de recréer l'ambiance et l'animation des lieux procuraient une vision du port profondément actuelle. Nulle métaphysique impériale, nulle image de permanence et d'immuabilité dans ces scènes grouillantes d'activité. Rien qui vienne affirmer la pérennité d'un régime monarchique. Les ports peints par Vernet sont des villes du dix-huitième siècle, des villes modernes dont le rôle vital se manifeste dans toute sa quotidienneté. Le désir de découvrir le monde qui prévalait à l'époque, la conscience économique naissante et la désuétude des symboles traditionnels n'incitaient pas à ennoblir artificiellement le paysage. De fait, Marigny aurait pu demander au peintre de représenter des ports antiques, ou de représenter les ports de France à l'antique. En choisissant plutôt une approche contemporaine et séculière, il souhaitait s'attirer les faveurs de la critique éclairée; mais peut-être ces ports parurent-ils trop incontrôlables aux yeux du monarque?

Dans une France qui avait fait du culte de Louis XIV une pierre d'assise de l'absolutisme, cette évolution de l'art officiel a pu surprendre Louis XV. Vernet a mis en scène une société apparemment libre et indifférente au sort du roi. Dans la représentation de la foule du port, le peintre s'est buté à un problème semblable à celui qu'avaient rencontré les commentateurs du Salon dans leur description du nouveau public ${ }^{59}$. En optant pour la multiplication des scènes et des types sociaux, Vernet se livrait à une description atomisante du peuple qui laissait deviner les clivages internes et contredisait l'idée abstraite qu'on aimait s'en faire. Trop concrets, cette société et ces paysages révélaient de façon manifeste l'absence du prince et affichaient une insolente indépendance à son égard. Peut-être n'apprécia-til pas de se voir si étranger en son royaume.

On pourrait rétorquer à cette analyse que le culte de la personnalité royale demeurait l'affaire de la peinture d'histoire et qu'il était donc normal que le roi soit ainsi évacué des paysa- ges de Vernet. Dans Le Portrait du roi ${ }^{60}$, Louis Marin prouve au contraire que tout dans l'art de la cour de Louis XIV célébrait la position centrale du monarque. Son chapitre intitulé " Le Roi et son géomètre " explique par exemple comment l'art de la cartographie, qui se voulait pourcant une science exacte disciplinée par l'objectivité instrumentale, utilisait justement cette caution scientifique pour confirmer la place du roi au centre géographique du royaume, doublant ainsi son centre politique. Sous Louis XV, l'art officiel n'est plus au seul service du culte royal; il véhicule même une vision de l'État qui s'en distancie. Si l'on admet que Louis XV ne s'est jamais préoccupé outre mesure de la marine, on constate que l'œuvre de Vernet n'a pas su entraîner la prise de conscience souhaitée par Marigny. Éloignés géographiquement de la métropole et de la tête de l'État, les ports semblent lui avoir étés tout aussi étrangers du point de vue symbolique.

Comme nous l'avons répété maintes fois, le Salon devint en ces années une arène convoitée. Le vingt-trois juillet 1759, après avoir reçu les deux vues du port de Bordeaux, Marigny écrivit à Vernet : "Ces deux tableaux rempliront une place, cette année, dans notre Salon, bien intéressante pour les connaisseurs, pour les amateurs et pour le public dont vous avez déjà mérité tant d'applaudissements. "161 Malheureusement pour l'auteur de ces lignes, la série des Ports faillit également dans sa tentative de séduire le public du Salon ${ }^{62}$. Nous en avons pour preuve les commentaires de la critique artistique, porte-parole auto-proclamé de la foule qui le fréquente ${ }^{63}$. Lors des présentations successives des Vues des ports au Salon, l'accueil fut en effet mitigé. D'emblée, il faut reconnaître que ces œuvres respectueuses des topographies locales et farcies de détails suscitèrent chez certains critiques un intérêt marqué. Ian Lochhead a recueilli et analysé différentes réactions de la critique devant les tableaux. Du Mercure de France de 1755, il tire ce commentaire élogieux sur le Port de Marseille (fig. 2) : "Vous y voyez ces ouvrages admirables, si utiles au commerce de la nation. Vous y voyez les habitants des quatre parties du mond [sic] réunis pour l'intérêt et le bien public agir, commercer ensemble. "64 À l'époque, comme aujourd'hui d'ailleurs, on appréciait les Ports de France pour leur caractère encyclopédique, car il est vrai qu'ils sont une mine de renseignements sur un univers peu connu des Parisiens. Nous ne sommes donc pas surpris de voir la critique louer l'abondance des informations techniques et les différentes scènes qui forment l'ensemble. Encore au Salon de 1755, un amateur anonyme ${ }^{65}$ applaudissait aux qualités pédagogiques des Ports: "Sur son port de Marseille et dans son arsenal de Toulon, dont les détails sont exacts, sans confusion et méthodiquement ordonnés, on apprendrait facilement des choses que bien des personnes devraient sçavoir, et dont elles auroient de la peine à se faire instruire. "66

Une telle lecture repose en partie sur le fait que malgré le 
point de vue éloigné qui embrasse le panorama, la finesse et la lisibilité des nombreux détails autorisent et même encouragent une plongée du regard du spectateur dans le tableau. Michael Fried attribue ce phénomène à la tension entretenue entre l'unité de la composition, assurée par la maîtrise de la perspective et l'harmonie lumineuse, et l'éclatement de cette unité d'ensemble par les oppositions formelles et la profusion des points d'intérêt :

I wish to suggest that we find often in Vernet's art, a tension or contradiction between, on the one hand, a subtle but forthright mode of decorative integration, capable of being taken in at a glance, and, on the other, a nerwork of rclationships among multiple disparate centers of interest whose separation from and connection with one another within an imaginary space can be apprehended only in time. ${ }^{67}$

Principes d'unité, la lumière et la perspective (d'où l'importance du point de vue) faciliteraient les transitions du regard entre les multiples détails disséminés dans le paysage, provoquant "l'absorbement " ${ }^{68}$ du regard du spectateur et son entrée fictive dans le tableau comme dans un paysage réel. Dans son Salon de 1763, Diderot invitait à contempler le Port de la Rochelle avec une lunette comme on le ferait en face d'un paysage réel. A propos de cette même œuvre, le critique écrivait ailleurs :

Le Port de la Rochelle est infiniment plus piquant. Voilà ce qu'on peut appeler un ciel; voilà des eaux transparcntes, et tous ces groupes, ce sont autant de petits tableaux vrais et caractéristiques du local; les figures en sont du dessin le plus correct. Comme la touche en est spirituelle et légère! Qui est-ce qui entend la perspective aérienne mieux que cet homme-là? ${ }^{69}$

Cet extrait permet de constater que Diderot admirait autant les traits d'ensemble (la perspective aérienne, la lumière du ciel et des eaux) que des éléments isolés (les tableaux formés par les groupes). Sans cet équilibre fragile, il n'y aurait vu sans doute qu'une plate accumulation de détails. On note également ce "local " qui fait partie du vocabulaire descriptif de Diderot et qui signifie qu'un tableau imite fidèlement la réalité.

C'est sous ce dernier angle que l'accueil de la cricique fut parfois plus froid. On ne peut certes parler d'un rejet pur et simple, mais on a vu s'exprimer des réserves et des commentaires qui s'engageaient davantage dans la voie de la théorie artistique. On reconnaissait bien des qualités pédagogiques et réalistes aux tableaux, mais jugés en tant qu'œuvres d'art, ils ne faisaient pas le poids. C'est que ces œuvres d'imitation, louables au demeurant pour leur réalisme, empêchaient le peintre d'exercer ses talents d'imagination et de création. Contraint par les prescriptions de L'Itinéraire, Vernet n'était pas libre d'interpréter le paysage et d'y ajouter une touche personnelle qui aurait fait d'une vue topographique une œuvre d'art ${ }^{70}$. Et si Lochhead dit remarquer une acceptation progressive du paysage réaliste (le paysage local de Diderot) au dix-huitième siècle, il concède dans le même souffle que le paysage d'imagination (idéal) demeurait le favori parce qu'il était le plus souvent historié et étoffé d'un sujet dramatique, comme dans le cas des tempêtes et des naufrages de Vernet. En optant audacieusement pour le paysage, Marigny cherchait à épargner pour un temps une peinture d'histoire meurtrie par une critique acrimonieuse et l'indifférence croissante du public du Salon. Ce fut peine perdue puisque malgré le recours à un peintre unanimement applaudi, la combinaison d'une esthétique réaliste et d'un message politique modernisé n'engendra qu'un compromis malhabile qui rata ses cibles.

En adoptant une perspective qui associe l'esthétique du temps, l'histoire sociale de l'art et l'histoire des mentalités, nous avons montré que le caractère conventionnel des marines de Vernet ne les empêchait pas d'être profondément inscrites dans l'actualité artistique de la moitié du dix-huitième siècle français et d'y tenir un rôle singulier. Globalement, les œuvres de Vernet que nous avons étudiées nous permettent de pénétrer la complexité de ce qu'on a appelé la montée du goût bourgeois. Celuici relève davantage de l'importance croissante du sentiment et de la subjectivité dans l'appréciation de l'œuvre d'art que d'une envie réelle d'entrer en contact avec la nature, ce qui peut expliquer la popularité des naufrages de Vernet et le moindre succès de la série des Ports. La faveur dont jouissait Vernet témoigne aussi de l'influence croissante du public (et de la critique) dans le cadre du Salon de l'Académie, influence qui signifie pour un nombre de plus en plus important d'artistes la possibilité de connaître une carrière flamboyante en dehors du cadre protégé de cette institution. En explorant enfin les modalités de la propagande officielle et en adaptant le schéma de Crow à la peinture de Vernet, nous avons enfin découvert que la crise de l'art officiel ne touchait pas que le genre historique, mais tenait plutôt au fonctionnement même de cette sphère artistique trop proche du politique. À vrai dire, l'étude de Vernet semble si riche que nous avouons notre étonnement devant le peu d'intérêt qu'a suscité son œuvre jusqu'à tout récemment.

Notes

L'auteur tient à exprimer sa reconnaissance envers Johanne Lamoureux pour son appui et ses conseils. Cet article est tiré d'un mémoire de maîtrise subventionné par le fonds FCAR.

1 Nous pensons par exemple aux écrits, par ailleurs très riches et innovateurs sur le plan théorique, de Michacl Fried, Absorption and Theatricality. Painting \& Beholder in the Age of Diderot, Berkeley, 
University of California Press, 1980, qui analyse audacieusement la peinture de Vernet, ou de Thomas Crow, Painters and Public Life in Eighteenth-Century Paris, New Haven, Yale University Press, 1985, qui la passe presque sous silence.

2 Il suffit de feuilleter la dernière décennie de Recherches sur Diderot et l'Encyclopédie pour s'en apercevoir.

3 Dans Fish Story, catalogue d'exposition, Rotterdam, Stockholm, Glasgow et Calais, 1995, Allan Sekula décrit très bien la disparition progressive de la mer de la culture occidentale au cours du vingtième siècle, et ce même si les ocćans remplissent toujours d'importantes fonctions économiques. Le succès du film Titanic de James Cameron (1997) et des destinations balnéaires fait tout sauf lever le voile, par exemple, sur les pratiques criminelles de nombreux armateurs.

4 Voir note 1 pour la référence complète.

5 Jacques Chouillet écrit que c'est ainsi que Diderot lui-même appelait ce passage du Salon de 1767 où deux promeneurs dialoguent dans un décor qui n'est en réalité qu'une suite de paysages présentés au Salon par Vernet; J. Chouillet, "La Promenade Vernet ", Recherches sur Diderot et l'Encyclopédie, no 2, avril 1987, 124-163.

6 Le traité, dont le titre complet est A Philosophical Enquiry into the Origin of Our Ideas of the Sublime and Beautiful, fut craduit en français en 1765, mais Diderot l'aurait peut-être lu en anglais en 1766; E. M. Bukdahl, Diderot, critique d'art, t. II, Copenhague, Rosenkilde et Bagger, 1982, 103 et 104.

7 Diderot renvoie sans détour au mot anglais astonishment, terme central dans l'histoire du concept du sublime.

8 Denis Diderot, Euvres, t. IV, Paris, Robert Laffont, 1996, 633.

9 Ibid.

10 Edmund Burke, A Philosophical Enquiry into the Origin of Our Ideas of the Sublime and Beautiful, Oxford et New York, Oxford University P'ress, 1990, 36.

11 Ibid., 54.

12 Diderot, op. cit., 357.

13 Le Salon de 1767 est célèbre pour son dialogue fictif qui met en scène deux protagonistes évoluant dans des paysages qui sont autant de tableaux de Vernet présentés au Salon cette année-là. Le passage que nous reprenons n'est pas tiré du dialogue, mais d'une autre description qui le suit immédiatement et qui évoque cette fois la narration d'un rêve.

14 Dideror, op. cit., 631.

15 C'est Lucrèce qui aurait exprimé pour la première fois le plaisir ressenti devant le spectacle du naufrage. Pour un complément d'information sur ce topos et sur son évolution aux 18 ème et 19 ème siècles, voir le texte de Carsten Zelle, "Théorie du naufrage : avant Géricault ", Géricault, t. I, Paris, La Documentation française, 1996, 179-225.

16 Elle soutient que l'émotion est souhaitée par Dideror en ce qu'elle forme un passage vers la connaissance: "Il (Diderot) désire que l'artiste interprète les motifs suggestifs dans le domaine de l'inquiétant, analysés par Burke, car le spectateur en les considérant peut se représenter les aspects tragiques et destructeurs de l'existence"; Bukdahl, op. cit., II, 108.

17 Bukdahl, Ibid., 106.
18 Herbert Dieckmann, Cing leçons sur Diderot, Genève et Paris, Minard et Librairie E. Droz, 1959, 111.

19 La confusion entretenue entre la nature et l'art, illustrée par la vogue des jardins anglais dits "naturels", mena d'ailleurs à sa conceptualisation dans la catégorie esthétique du pittoresque.

20 De la même façon qu'il attribue à Rousseau la vogue des voyages en Suisse, Daniel Mornet avance l'hypothèse que "ce fut Vernet qui mit à la mode les tempêtes et les clairs de lune, les abîmes mugissants et les montagnes qui haussent d'inaccessibles sommets "; Mornet, Le Sentiment de la nature en France. De J.J. Rousseau à Bernardin de Saint-Pierre, New York, Burt Franklin, 1971, 340.

21 Son Robinson Crusoë (1719) bien sûr, mais aussi une volumineuse Histoire générale des plus fameux Pyrates, I et II, publiée en 1726, rééditée à Paris, Phebus, 1990, et dont on vient tout récemment de lui attribuer la paternité.

22 Loin d'être refoulée, la peur se manifesta en fait de façon plus sensationnelle dans la littérature anglaise. Dans un article sur les récits de naufrages, Monique Brosse remonce à l'apparition de ce genre qui connaîtra un vif succès au dix-neuvième siècle: "Au XVIIle siècle, en Angleterre surtout, la presse commence à publier, sporadiquement, de brefs échos de naufrages "; Monique Brosse, "Littérature marginalc : les histoires des naufrages", Romantisme, no 4, 1972, 112.

23 Jean Delumeau, La Peur en Occident XIVe-XVIIIe siècles, Paris, Fayard, 1978, 41.

24 T.S.R. Boase, "Shipwrecks in English Romantic Painting ", Journal of the Warburg and Courtauld Institutes, no 22, 1959, 332.

25 Alain Corbin, Le Territoire du vide. L'Occident et le désir du rivage (1750-1840), Paris, Aubier, 1988. Remarquons que les naufrages de Vernet ont toujours lieu sur la côte et qu'on ne trouve nulle représentation de la haute mer dans son ceuvre.

26 Il faut insister sur le fait que l'expérience de la mer était rarissime chez. les contemporains de Diderot, et que le critique lui-même ne l'aurait aperçue pour la première fois qu'en 1773, soit bien des années après avoir écrit sur les marines de Vernet; voir Roland Desmé, "Diderot et la mer ", La Mer au siècle des encyclopédies, actes du colloque de Brest, Paris et Genève, Champion-Slatkine, $1987,103$.

27 Corbin, op. cit., 12.

28 Ibid., 14.

29 Ibid., 12.

30 Pour une analyse des significations morales, politiques ou religieuses attribuées à la mer dans la peinture de marine hollandaise du dix-septième siècle, voir L.O. Goedde, Tempest and Shipwreck in Dutch and Flemish Art: Convention, Rhetoric, and Interpretation, University Park, Pennsylvania State University, 1989.

31 Pour plus de détails concernant ces questions juridiques, consulter sur le site Internet de la Revue Juridique NEPTUNUS l'article de François-Xavier Pierronnet, "Le Droit de pense Ouessantin aux XVIIe-XIXe siècles: Entre législation du bris et tradition insulairc ", Neptunus, II et III (1997); adresse électronique: http:// www.droit.univ-nantes. fr/labos/cdmo/nept/nept.htm.

32 Le Grand Tour était un voyage initiatique de quelques années que 
les jeunes Anglais de la gentry effectuaient en Italie pour s'initier aux cultures romaine et de la Renaissance. Ce n'est que plus tard, sous l'impulsion de Gilpin, que le voyage pittoresque, une version allégée et raccourcie du Grand Tour, connut une véritable popularité

33 On attendait encore l'invention d'une horloge véritablement précise et fiable, instrument essentiel pour la détermination de la longitude, ce qui obligeait les capitaines à de savants calculs que tous ne maîtrisaient pas, ou à naviguer à vue en suivant la côte traîtresse.

34 Jean Ehrard, L'Idée de nature en France dans la première moitié du XVIIIe siècle, Genèvc et Paris, Slatkinc, 1981.

35 À la même époque plus au Sud, la chaude Méditerranée restera plus longtemps en disgrâce parce qu'on considérait cette mer bordéc de marais comme un environnement malsain. Si l'on se baignait parfois dans ses eaux, c'était avec une certaine répugnance et avec l'impression coupable de se livrer à une accivitć des classes inférieures qui reste malheureusement peu documentée

36 On rappellera à propos de cetce conception cénesthésique de la maladie que Burke liait intimement sentiment et physiologie dans son traité du sublime.

37 Corbin, op. cit., 76.

38 Dans Ideology of the Aesthetic, Cambridge, Basil Blackwell, 1990, 54, Terry Eagleton tente de démontrer que la société bourgeoise libérale se fonde depuis ses débuts sur des critères d'ordre esthétique. Sa cohésion dépendrait ainsi du concept du beau, principe de séduction et agent de la libre adhésion à la communauté, alors que sa vitalité proviendrait d'un concept du sublime associé à l'individualisme et à la compétition qui incarnent les valeurs du progrès : "The sublime is the anti-social condition of all sociability ". Moins ambitieuse, notre construction idéologique du sublime en fait plutôt un facteur de nivellement qui reprend en termes esthétiques la pensée universaliste en vogue dans certains cercles progressistes du dix-huitième siècle.

39 À la décharge des autorités de l'Académie, il faut reconnaître que le discours et la pensée artistiques de la moitié du dix-huitième siècle sont imprégnés de la hiérarchie des genres à un tel point que même la critique la plus progressiste ne peut éviter de l'intégrer à sa grille d'analyse. Sur ce sujet, voir le livre de Richard Wrigley, The Origins of French Art Criticism. From the Ancien Regime to the Restoration, Oxford, Oxford University Press, 1993.

40) La représentation d'un navire en pleine mer n'aurait pourtant pas constitué un précédent. La peinture hollandaise nous montre en effet souvent des bateaux seuls au milieu de l'étendue d'eau; le point de vue devient alors celui de Dieu mettant l'humanité au défi.

41 Malgré l'allure factice et conventionnelle de ces paysages, la lecture de la critique laisse supposer qu'on leur trouvait une facture fidèle à la réalité.

42 Sur la vocation mercantile des ateliers de peinture, voir Jean Chatelus, Peindre à Paris au XVIIIe siècle, Nîmes, Éditions J. Chambon, 1991

43 Léon Lagrange, Joseph Vernet et la peinture de marine au XVIIle siècle, Paris, Librairie Académique Didier, 1864.
44 Florence Ingersoll-Smouse, Joseph Vernet, peintre de marine 17141789: Étude critique suivie d'un catalogue raisonné de son æuvre peint avec 357 reproductions, Paris, Étienne Bignou, 1926.

45 Cette idée qu'on retrouve entre autres chez Louis Marin (voir note 60) et David Castriota (voir note 46) prend sa source dans Les Deux corps du roi: Essai sur la théologie politique au Moyen Âge, Paris, Gallimard, 1989, d'Ernst Kantorowicz, ouvrage qui traite de l'existence physique et juridique du roi dans l'Angleterre médiévale.

46 David Castriota, Artistic Strategy and the Rhetoric of Power. Political Uses of Art from Antiquity to the Present, Carbondale, Southern Illinois University Press, 1986, 8.

47 Ibid.

48 Selon Fernand Braudel, la lenteur des communications de l'époque faisait de la France un immense pays, un "monstre " de la stature d'un empire dans l'Europe pré-révolutionnaire; Braudel, L'Identité de la France, tome I: Espace et Histoire, Paris, ArthaudFlammarion, 1986, 279.

49 W.J.T. Mitchell, Landscape and Power, Chicago, The Univcrsity of Chicago Press, 1994, 14.

50 Ibid., 15.

51 Cette remarque est empruntée à Philip Conisbee, Claude-Joseph Vernet, 1714-1789, catalogue de l'exposition du Greater London Council (4 juin au 19 septembre 1976) et du Musée de la Marine de Paris (14 octobre 1976 au 9 janvier 1977), notice no. 35.

52 Braudel, op. cit., II, 247

53 Braudel, op. cit., I, 35.

54 "A côté de l'administration monarchique, l'armée est donc devenue l'outil le plus actif de la formation unitaire de la France." Ibid., 339.

55 En plus des raisons susmentionnées, on peut imputer le fait que Marigny semble avoir pris seul l'initiative des Ports au manque de panache des Rouillé de Jouy (1749-1753) et Machault d'Arnouvelle (1753-1756), qui ont occupé le poste de ministre de la marine pendant cette période; Claude Farrère, Histoire de la Marine française, Paris, Flammarion, 1962.

56 Crow, op. cit., 113.

57 Nous ignorons la date et les circonstances exactes de cette installation au Luxembourg, mais à la lecture de Manœuvre, on peut croire qu'elle s'est faite du vivant de Louis XV : "Louis XV aime la chasse qui, indubitablement, l'intéresse plus que la marine. Au contraire des représentations des chiens favoris du monarque, les vues des ports de France n'ont pas trouvé place dans les palais royaux, Versailles, Fontainebleau ou Compiègne. Elles sont relégués au Luxembourg. "Laurent Manœuvre et Eric Rieth, Joseph Vernet 1714-1789. Les Ports de France, Arcueil, Anthèse, 1994, 16.

58 En pleine période rococo, on peut lier ce rejet au fait que Louis XV n’aimait pas la peinture "sérieuse", comme le fait Laurent Manœuvre dans l'introduction de son livre consacré aux Ports de France. C'est plausible, mais le roi n'avait-il pas soutenu l'entreprise de Marigny durant onze années?

59 Sur ce sujet, voir l'introduction de Crow, qui présente avec intelligence la difficulté que constitue pour les commentateurs du Salon 
la description du public comme une entité homogène, sa principale caractéristique étant justement de renfermer une grande diversité de types sociaux.

60 Louis Marin, Le Portrait du roi, Paris, Minuit, 1981.

61 Le Port des Lumières. La peinture à Bordeaux, 1750-1800, catalogue d'exposition, Bordeaux, Musée des Beaux-Arts de Bordeaux, 1989, 36.

62 Concédons qu'il serait malhonnête de parler d'un véritable échec. Si les commentaires de la critique ne sont pas aussi élogieux que dans le cas des autres productions de Vernet, le succès commercial remporté par les estampes tirécs de la série des Ports de France démontre que l'accueil du public fut certainement favorable.

63 Pour de plus amples réflexions sur la question complexe du caractère représentatif de la critique artistique du Salon de l'Académie, nous ne pouvons que renvoyer à l'introduction du livre de Crow et à l'ouvrage de Wrigley.

64 Ian J. Lochhead, The Spectator and the Landscape in the Art Criticism of Diderot and his Contemporaries, Ann Arbor, University of Michigan Research Press, 1981, 38.

65 D'après Conisbee, il s'agirait de Delaporte, l'auteur des Sentiments sur plusieurs tableaux exposés cette année dans le grand Salon du Louvre; Conisbee, op. cit., no. 34.

66 Lochhead, op. cit., 38.

67 Fried, op. cit., 134.

68 Dans son livre, Fried réfléchit sur ce qu'il appelle l'entrée fictive du spectateur dans le tableau, ce qui entraîne la disparition de la distance entre la réalité et l'œuvre d'art et conséquemment la négation de sa nature artistique. Le choix du terme " absorbement " est celui du traducteur de Fried chez Gallimard.

69 Diderot, Euvres esthétiques, éd. Paul Vernière, Paris, Garnier, 1968, 564.

70 On pourrait critiquer notre rapprochement de cette série avec les autres marines de Vernet sous prétexte que les Vues de ports ne seraient pas des œuvres d'art, mais bien des vues topographiques relevant davantage de la cartographie. Nous répondrons que leur présentation au Salon de l'Académie et le fait que Diderot inscrive son commentaire sur les Ports en continuité avec ses autres critiques, autorisent à les considérer comme des œuvres d'art à part entière qui répondent à des critères esthétiques au même titre que les naufrages de l'artiste. 